\title{
DEGRADASI ZAT WARNA SUDAN I SECARA SONOLISIS DAN FOTOLISIS DENGAN PENAMBAHAN TiO ${ }_{2}$-ANATASE
}

\author{
Safni $^{1,3}$, Umiati Loekman ${ }^{1}$, Fitra Febrianti ${ }^{1}$, Maizatisna ${ }^{1}$, Tadao Sakai ${ }^{2}$ \\ ${ }^{1}$ Jurusan Kimia FMIPA Universitas Andalas, Padang \\ ${ }^{2}$ Department of Chemistry, Aichi Institute of Technology Toyota City, Japan \\ 3email : safni@yahoo.com
}

\begin{abstract}
Degradation of Sudan I dye had been done with sonolysis and photolysis by adding anatase- $\mathrm{TiO}_{2}$. Sonolysis method were performed using an ultrasound with frequency of $45 \mathrm{kHz}$. Photolysis method were performed using an irradiation of UV light with $\lambda=359 \mathrm{~nm}$. Degradation of $4 \mathrm{mg} / \mathrm{L}$ Sudan I with addition of $0.1000 \mathrm{~g}$ anatase- $\mathrm{TiO}_{2}$ at $\mathrm{pH}=7.0$, temperature $25 \pm 1{ }^{\circ} \mathrm{C}$, percentage of degradation $64.04 \%$ within 180 minutes sonolysis. Degradation of $4 \mathrm{mg} / \mathrm{L}$ Sudan I using photolysis with addition of $0.1000 \mathrm{~g}$ anatase- $\mathrm{TiO}_{2}$ at $\mathrm{pH}=7.0$ and percentage of degradation $100 \%$ within 180 minutes photolysis. Degradation of $4 \mathrm{mg} / \mathrm{L}$ Sudan I using sonolysis and photolysis simultaneously with addition of $0.1000 \mathrm{~g}$ anatase- $\mathrm{TiO}_{2}$ at $\mathrm{pH}=7.0$ and percentage of degradation $94.04 \%$ within 180 minutes treatment.
\end{abstract}

Keywords : degradation, sonolysis, photolysis, Sudan I, anatase-TiO ${ }_{2}$.

\section{DAFTAR PUSTAKA}

1. Andayani, W., Sumartono, A., 1999, Aplikasi Radiasi Pengion Dalam Penguraian Limbah Industri, Radiolisis Larutan Standar Zat Warna Reaktif Cibarcon Violet 2R, Majalah Batan, Vol. XXXII No. $1 / 2$.

2. Destaillats, H., Anderson, T. W., Hoffmann, M. R., 2001, Aplication of Ultrasound in NAPL Remediation Sonochemical Degradation of TCE in Aqueous Surfactant Solutions, J. Environ. Sci. Technol.,35: 3019-3024.

3. http://www.und.ac.za/und/org/sonochem. 5 Januari 2006).

4. http://www.KimiaUI.titania/2000/htm.Akti vitas Fotokatalitik pada Permukaan $\mathrm{TiO}_{2}$. (10 Januari 2006).

5. Stock, N. L., Peller, J., Vinadgopal, K., Kamat, P. V., 2000, Combinative Sonolysis and Photocatalysis for Textile Dye Degradation, J. Environ. Sci. Technology., 34: 1747-1750.

6. Hiskia, A., Ecke, M., Troupis, A., Kokorakis, A., Hennig, H., and Papaconstantnou, E., 2001, Sonolytic,
Photolytic and Photocatalytic Decomposition of Atrazine in the Presence of Polyoxometalates, J. Environ. Sci. Techology., 35: 2358-2364.

7. http://en.Wikipedia.org/wiki/SudanI. (2 Januari 2006).

8. http://www.saujaya.com/sol_yel14.htm. (5 Januari 2006).

9. http://ntp.niehs.nih.gov/index.SUDANI. (2 Januari 2006).

10. http://ww.ncbi.nlm.nih.gov/entrez/query. (2 Januari 2006).

11. Arief, S., Safni, Roza, P. P., 2007, Degradasi Senyawa Rhodamin B Secara Sonolisis dengan Penambahan $\mathrm{TiO}_{2}$ Hasil Sintesa Melalui Proses Sol-Gel, J. Ris. Kim., 1(1): 64-70.

12. Peller, J., Wiest, O., Kamat, P. V., 2001, Sonolysis of 2,4-Dichlorophenoxyacetic Acid in Aqueous Solution. Evidence for OH- Radical-Mediated Degradation, $J$. Phys. Chem. A., 105: 3176-3181.

13. Basri, D., 2005, Degradasi Metilen Biru Secara Sonolisis dengan Penambahan $\mathrm{TiO}_{2}$-Anatase, Skripsi, Universitas Andalas.

14. Wang, J., Guo, B., Zhang, X., Zhang, Z., Han, J., and Wu, J., 2005, Sonocatalytic 
Degradation of Methyl Orange in the Presence of $\mathrm{TiO}_{2}$ Catalysts and Catalytic Activity Comparison of Rutile and Anatase, J. Ultrasonics Sonochemistry., 12: 331-337.

15. Khopkar, S. M., 1990, Konsep Dasar Kimia Analitik, UI Press, Jakarta, 201-227.
16. Safni, Zuki, Z., Haryati, C., 2008, Degradasi Zat Warna Alizarin S Secara Sonolisis dan Fotolisis Dengan Penambahan $\mathrm{TiO}_{2}$-anatase, $J$. Pilar, (submitted). 